\title{
Thermophoresis of a slightly deformed aerosol sphere
}

\author{
Sergey Senchenko and Huan J. Keh ${ }^{\text {a) }}$ \\ Department of Chemical Engineering, National Taiwan University, Taipei 10617, Taiwan, Republic of China
}

(Received 4 September 2006; accepted 1 February 2007; published online 27 March 2007)

\begin{abstract}
An analytical study is presented for the thermophoretic motion of a freely suspended aerosol particle with an arbitrary, slightly deformed spherical surface in a uniformly prescribed temperature gradient. The Knudsen number is assumed to be small so that the fluid flow is described by a continuum model with a temperature jump, a thermal slip, and a frictional slip at the surface of the particle. A first attempt is made to obtain analytical approximations for the thermophoretic velocity of the particle in the limit of vanishing Peclet and Reynolds numbers. To the first order in the small parameter characterizing the deformation of the spherical shape of the particle, explicit expressions are derived for its drift and rotational velocities. The angular velocity of a particle undergoing thermophoresis is found to be zero for any shape, which is the first indication that the fluid motion around a single thermophoretic particle of an arbitrary shape is irrotational, as is known to be in the case of thin-double-layer electrophoresis. A comparison of our first-order approximation for the thermophoretic velocity of a spheroid with the available exact solution shows that the agreement is quite good, even for relatively large deformations of the spherical shape of the particle. Our results for the motion of a spheroid demonstrate that its relative physical and surface properties, its aspect ratio, and its orientation relative to the imposed temperature gradient can have significant effects on its thermophoretic mobility. (C) 2007 American Institute of Physics. [DOI: 10.1063/1.2712902]
\end{abstract}

\section{INTRODUCTION}

Thermophoresis refers to the motion of aerosol particles in response to a temperature gradient. This phenomenon was first described in 1870 by Tyndall, who observed a dust-free zone in a dusty gas around a hot body. ${ }^{1}$ The thermophoretic effect can be explained in part by appealing to the kinetic theory of gases. ${ }^{2}$ The higher energy molecules in the hot regions of the gas impinge on the particle with greater momenta than molecules coming from the cold regions, thus resulting in the migration of the particle in the direction of decreasing temperature. Being a mechanism for the capture of aerosol particles on cool surfaces, thermophoresis is of considerable importance in many practical applications, such as sampling of aerosol particles, ${ }^{3}$ cleaning of air, ${ }^{4}$ scale formation on surfaces of heat exchangers, ${ }^{5}$ modified chemical vapor deposition, ${ }^{6,7}$ manufacturing of microelectronics, ${ }^{8}$ nuclear reactor safety, ${ }^{9}$ and removal of soot aerosol particles for combustion exhaust gas systems. ${ }^{10}$

It is convenient to express the thermophoretic velocity of a particle in a constant temperature gradient $\nabla T_{\infty}$ as

$$
\mathbf{U}=-M \nabla T_{\infty}
$$

where the negative sign indicates that the motion is in the direction of decreasing temperature. The thermophoretic mobility $M$ depends on the magnitude of the Knudsen number, $l / a$, where $a$ is a characteristic linear dimension of the particle and $l$ is the mean free path of the gas molecules.

In the regime of small Knudsen number where the particle is large compared with the mean free path, the fluid flow may be described by a continuum model and the thermo-

${ }^{a)}$ Electronic mail: huan@ntu.edu.tw phoretic force arises from an induced thermal slip along the particle surface due to the existence of a tangential temperature gradient at the particle-fluid interface. Utilizing the gas kinetic theory, Maxwell ${ }^{11}$ predicted that a tangential temperature gradient $\nabla_{s} T$ at a gas-solid interface would cause a thin layer of gas (known as the Knudsen layer) adjacent to the surface to move (which can be viewed as a thermo-osmotic flow), with the relative velocity at the outer edge of the layer being

$$
\mathbf{v}_{s}=\frac{C_{s} \mu}{\rho T_{0}} \nabla_{s} T,
$$

where $\mu$ is the fluid viscosity, $\rho$ is the fluid density, and $T$ is the local gas temperature. The thermal slip coefficient $C_{s}$ was found to be 3/4 by Maxwell on the assumption that the distribution function in the bulk of the gas held all the way to the solid wall. The thermal slip velocity $\mathbf{v}_{s}$ is directed toward the high temperature side.

By using Eq. (2), which gives the coupling between temperature and velocity fields, as a slip velocity boundary condition and solving the equation of fluid motion at low Reynolds number incorporating with the heat conduction in the gas and particle, Epstein ${ }^{12}$ derived the following formula for the thermophoretic mobility of a suspended aerosol sphere:

$$
M=\frac{C_{s} \mu}{\rho T_{0}} \frac{2 k_{f}}{2 k_{f}+k_{p}},
$$

where $k_{f}$ and $k_{p}$ are the thermal conductivities of the gas and particle, respectively, and $T_{0}$ is the bulk-gas absolute temperature at the particle center in the absence of the particle (or the mean gas temperature in the vicinity of the particle). On the other hand, in a new way of describing thermophore- 
sis, Brenner and Bielenberg ${ }^{13}$ recently suggested that the thermophoretic movement of aerosol particles occurs because of a nonzero volume-averaged velocity that exists in a continuum subjected to a density gradient, even though mass-average motion is absent. Without involving Maxwell's thermally induced velocity-slip condition given by Eq. (2) or free phenomenological parameters such as $C_{s}$, they obtained an analytical expression for the thermophoretic velocity of a spherical particle which agrees with Eq. (3). The thermophoretic mobility predicted by Eq. (3), which is independent of particle size, is in fair agreement with experimental data when the ratio $k_{p} / k_{f}$ is not too high, less than about 10 , as for oil droplets. But it gives too small thermophoretic velocities (by a factor 30 or more) for particles with large thermal conductivity, for instance, $\mathrm{NaCl}$ (sodium chloride) particles with $k_{p} / k_{f} \approx 100$. Indeed, in the limit $k_{p} / k_{f} \rightarrow \infty$, Eq. (3) yields $M=0$, whereas Schadt and Cadle ${ }^{14}$ distinctly observed and measured a thermophoresis with $\mathrm{NaCl}$ particles.

Equation (3) for the thermophoretic mobility of an aerosol sphere was improved by Brock ${ }^{15}$ using the low-Knudsennumber effects of temperature jump at the particle surface as well as frictional (isothermal) gas slippage (in addition to the thermal slip velocity) along the particle surface. The resulting expression for the thermophoretic mobility of the aerosol sphere is

$$
M=M^{*}=\frac{C_{s} \mu}{\rho T_{0}} \frac{2\left(k_{f}+k_{p} C_{t} l / a\right)}{\left(1+2 C_{m} l / a\right)\left(2 k_{f}+k_{p}+2 k_{p} C_{t} l / a\right)},
$$

where the dimensionless coefficients $C_{t}$ and $C_{m}$ account for the temperature jump and frictional slip, respectively, at the particle surface and must be determined experimentally for each gas-solid system. Note that Eq. (4) is applicable to the range of finite Knudsen number and reduces to Eq. (3) when $l / a=0$. For large particles with $k_{p} / k_{f} \rightarrow \infty$, Eq. (4) yields $M$ $=2\left(C_{s} \mu / \rho T_{0}\right)\left(C_{t} l / a\right)$, whereas the Epstein formula (3) gives no particle migration.

Derjaguin et al. ${ }^{16}$ presented the experimental data of thermophoretic velocities for a variety of aerosols, which are in good agreement with Eq. (4) with $C_{m}=0, C_{s}=3 / 2$, and suitable selection of coefficient $C_{t}$. Later, the thermophoretic force on aerosol spheres was measured over a wide range of Knudsen numbers and particles' thermal conductivities. ${ }^{17}$ It was also found that the data in the slip-flow regime agree with Eq. (4). A set of kinetic-theory values for complete thermal and momentum accommodations appear to be $C_{s}$ $=1.17, C_{t}=2.18$, and $C_{m}=1.14 .{ }^{18}$ Recently, kinetic-theory values of these slip coefficients have been obtained accurately under various conditions. ${ }^{19-23}$ According to Eq. (4), particles with large thermal conductivity and small Knudsen number (say, $k_{p} / k_{f}=100$ and $l / a=0.01$ ) will migrate by thermophoresis at velocities of $10-50 \mu \mathrm{m} / \mathrm{s}$ in temperature gradients of order $100 \mathrm{~K} / \mathrm{cm}$, such gradients are easily attainable in thermal boundary layers.

Most aerosol particles are not spherical ${ }^{24,25}$ and it is therefore of considerable interest to examine the effect of particle shape on thermophoresis. The thermophoretic theories of nonspherical particles differ from those of spheres in that there is an orientational problem. In general, the thermo- phoretic mobility of a nonspherical particle is anisotropic and the velocity of the particle is no longer collinear with the prescribed temperature gradient. In the purely continuum approach for the thermophoretic velocity containing no free parameters, ${ }^{13}$ the effect of shape and orientation of nonspherical particles is briefly discussed, with specific results furnished for effectively nonconducting particles. The thermophoretic motion of a long circular cylinder in the direction normal to its axis has been studied with the consideration of the effects of temperature jump, thermal slip, and frictional slip at the particle surface. ${ }^{26,27}$ On the other hand, the axisymmetric thermophoresis of a spheroidal particle along its axis of revolution with thermal slip but without temperature jump and frictional slip at its surface was also analyzed. ${ }^{28,29}$ Recently, this analysis has been generalized to a spheroid with an arbitrary orientation relative to the thermal gradient. ${ }^{30}$ However, the problem of thermophoresis of spheroidal particles with all the effects of temperature jump, thermal slip, and frictional slip at the particle surface has not been solved yet, mainly due to the fact that, if the temperature jump and/or frictional slip is included, a separable solution is not feasible for most orthogonal curvilinear coordinate systems, such as the prolate and oblate spheroidal ones.

In this work we analyze the movement of a rigid particle with an arbitrary, slightly deformed spherical shape immersed in a gaseous medium with a prescribed uniform temperature gradient, and the temperature jump, thermal slip, and frictional slip boundary conditions prevail at the particle surface. The object is to obtain a correction to Eq. (4) for the thermophoretic mobility of the slightly deformed spherical particle. In Sec. II, we formulate the problem of the thermal and hydrodynamic fields around a particle with an arbitrary shape undergoing thermophoresis and specify the boundary conditions. The expansions for the temperature and fluid velocity distributions in power series of a small parameter characterizing the deviation of a slightly deformed sphere from the spherical shape are introduced in Sec. III, where formal expressions for the first correction to the unperturbed boundary conditions are derived. Section IV briefly recapitulates the structure of the unperturbed temperature and flow fields as well as thermophoretic velocity, while Sec. V treats the lowest-order perturbation of the thermal and hydrodynamic fields due to the deviation from the spherical shape of the particle. In Sec. VI, we derive a general expression for the thermophoretic velocity of the slightly deformed spherical particle. Finally, in Sec. VII, we consider some special cases of interest, viz., the thermophoresis of prolate and oblate spheroids, compare the results of our analytical approximation with the exact solution obtained for thermophoretic spheroids with $C_{m}=C_{t}=0$, and discuss some relevant physical characteristics of the thermophoretic motion of spheroidal particles.

\section{BASIC EQUATIONS FOR THE THERMOPHORETIC MOTION OF AN ARBITRARY PARTICLE}

Consider a rigid aerosol particle with an arbitrary shape and a small Knudsen number undergoing thermophoresis in an unbounded incompressible Newtonian fluid of viscosity 
$\mu$. The Knudsen layer is assumed to be thin and the fluid may slip thermally and frictionally at the surface of the particle. The ensuing fluid velocity and pressure fields are denoted by $\mathbf{v}$ and $p$, respectively. Thus, the fluid motion at small Reynolds numbers is governed by the Stokes equations

$$
\mu \Delta \mathbf{v}=\boldsymbol{\nabla} p, \quad \boldsymbol{\nabla} \cdot \mathbf{v}=0
$$

subject to a particular set of boundary conditions. The general solution of the Stokes equations is given by ${ }^{31}$

$$
\begin{aligned}
\mathbf{v} & =\sum_{n}\left(\boldsymbol{\nabla} \varphi_{n}+\boldsymbol{\nabla} \times\left(\mathbf{r} \chi_{n}\right)+\frac{n+3}{2 \mu(n+1)(2 n+3)} r^{2} \boldsymbol{\nabla} p_{n}\right. \\
& \left.-\frac{n}{\mu(n+1)(2 n+3)} \mathbf{r} p_{n}\right),
\end{aligned}
$$

where $\varphi_{n}, p_{n}$, and $\chi_{n}$ are solid spherical harmonics of order $n, \mathbf{r}$ is the position vector relative to the particle center, and $r=|\mathbf{r}|$.

Let the particle have a thermal conductivity $k_{p}$ and the surrounding fluid have a thermal conductivity $k_{f}$; both are assumed to be constant. The Peclet number for the heat transfer in the fluid is assumed to be small; hence, the energy equations governing the temperature distributions are

$$
\Delta T_{f}=0 \quad \text { and } \quad \Delta T_{p}=0
$$

for the fluid and particle, respectively. For the temperature fields we perform a change of variables,

$$
T_{f}=\Theta+T_{0}+\mathbf{G} \cdot \mathbf{r}, \quad T_{p}=\Psi+T_{0}+\mathbf{G} \cdot \mathbf{r},
$$

where the constants $T_{0}$ and $\mathbf{G}$ prescribe the linear temperature field in the fluid at infinity and the thermal fields $\Theta$ and $\Psi$ are also governed by the Laplace equation.

The thermal boundary conditions at the particle surface require that the normal heat fluxes be continuous and a temperature jump which is proportional to the normal temperature gradient ${ }^{2,15}$ occur. Also, the fluid temperature must approach the linear prescribed field far from the particle and the temperature field inside the particle is finite everywhere. Thus, we have

$$
\begin{aligned}
& \left.k_{p} \mathbf{n} \cdot(\boldsymbol{\nabla} \Psi+\mathbf{G})\right|_{S}=\left.k_{f} \mathbf{n} \cdot(\boldsymbol{\nabla} \Theta+\mathbf{G})\right|_{S}, \\
& \left.(\Theta-\Psi)\right|_{S}=\left.C_{t} l \mathbf{n} \cdot(\boldsymbol{\nabla} \Theta+\mathbf{G})\right|_{S}, \\
& \left.\Psi\right|_{r=0}<\infty, \\
& \left.(\Theta / r)\right|_{r \rightarrow \infty} \rightarrow 0,
\end{aligned}
$$

where $\mathbf{n}$ is the unit normal vector at the surface of the particle, $l$ is the mean free path of a gas molecule, $C_{t}$ is the coefficient of the temperature jump, and $S$ denotes the local position at the particle surface. In the present analysis, Eq. (12) can also be written as $\left.\Theta\right|_{r \rightarrow \infty} \rightarrow 0$; however, this fine distinction may become relevant in other extensions, such as to asymptotic expansions in the context of small convective transport effects. Similar to the velocity field expressed as Eq. (6), $\Theta$ and $\Psi$ can be presented as sums of solid spherical harmonics,

$$
\Theta=\sum_{n} \Theta_{n}, \quad \Psi=\sum_{n} \Psi_{n} .
$$

We assume that the particle translates with the constant thermophoretic velocity $\mathbf{U}$ and rotates with the angular velocity $\boldsymbol{\Omega}$. Owing to the thermal and frictional slip velocities along the solid-fluid interface, the boundary condition for the fluid velocity at the particle surface is ${ }^{15,32}$

$$
\begin{aligned}
\left.\mathbf{v}\right|_{S}= & \left(\mathbf{U}+\mathbf{\Omega} \times \mathbf{r}+\frac{C_{m} l}{\mu} \mathbf{P}_{\tau}(\mathbf{v})\right. \\
& \left.+\frac{C_{s} \mu}{\rho T_{0}}\left(\nabla_{s} \Theta+\nabla_{s}(\mathbf{G} \cdot \mathbf{r})\right)\right)\left.\right|_{S}
\end{aligned}
$$

and the fluid flow vanishes far away from the particle,

$$
\left.\mathbf{v}\right|_{r \rightarrow \infty}=\mathbf{0} .
$$

Here $C_{s}$ is the thermal slip coefficient defined in Eq. (2), $C_{m}$ is the frictional slip coefficient, $\boldsymbol{\nabla}_{s} T=\boldsymbol{\nabla} T-(\boldsymbol{\nabla} T \cdot \mathbf{n}) \mathbf{n}$ and $\mathbf{v}$ is expressed by Eq. (6). The tangential stress vector acting along the surface of the particle is given by

$$
\mathbf{P}_{\tau}(\mathbf{v})=\boldsymbol{\sigma}(\mathbf{v}) \cdot \mathbf{n}-(\mathbf{n} \cdot \boldsymbol{\sigma}(\mathbf{v}) \cdot \mathbf{n}) \mathbf{n},
$$

where $\boldsymbol{\sigma}(\mathbf{v})$ is the deviatoric stress tensor.

\section{BOUNDARY CONDITIONS IN EXPANSION FORMS FOR A THERMOPHORETIC SPHERE WITH A SLIGHTLY PERTURBED SURFACE}

We now consider the thermophoretic motion of a slightly deformed spherical particle subject to a uniform temperature gradient G. It is assumed that the shape of the particle in spherical coordinates $(r, \theta, \phi)$ reads

$$
r=a(1+\varepsilon f(\theta, \phi))
$$

where $\varepsilon$ is a dimensionless parameter whose magnitude is much smaller than unity and $f(\theta, \phi)$ is an arbitrary function of angular position which is of order unity with respect to $\varepsilon$. The expansions for the velocity and temperature fields naturally acquire the structure

$$
\begin{aligned}
& \mathbf{v}=\sum_{i=0}^{\infty} \varepsilon^{i} \mathbf{v}_{i}, \quad \mathbf{U}=\sum_{i=0}^{\infty} \varepsilon^{i} \mathbf{U}_{i}, \quad \boldsymbol{\Omega}=\sum_{i=0}^{\infty} \varepsilon^{i} \boldsymbol{\Omega}_{i}, \\
& \Psi=\sum_{i=0}^{\infty} \varepsilon^{i} \Psi^{(i)}, \quad \Theta=\sum_{i=0}^{\infty} \varepsilon^{i} \Theta^{(i)} .
\end{aligned}
$$

Obviously, each $\mathbf{v}_{i}$ must itself satisfy the Stokes equations, while $\Theta^{(i)}$ and $\Psi^{(i)}$ are the solutions of the Laplace equation.

Using the expression for the unit normal vector on the particle surface $\mathbf{n}=\mathbf{r} / r-\varepsilon a \boldsymbol{\nabla} f$, we can expand the boundary condition (14) in successive powers of $\varepsilon$ by a Taylor series expansion about the unperturbed geometry, which corresponds to the spherical surface of $r=a$. The resulting conditions in the leading order are 


$$
\begin{gathered}
\left.\left(\mathbf{v}_{0}-\frac{C_{m} l}{\mu} \mathbf{P}_{\tau 0}\right)\right|_{r=a} \\
=\mathbf{U}_{0}+\mathbf{\Omega}_{0} \times a \frac{\mathbf{r}}{r}+\frac{C_{s} \mu}{\rho T_{0}}\left(\mathbf{G}-\left(\mathbf{G} \cdot \frac{\mathbf{r}}{r}\right) \frac{\mathbf{r}}{r}\right) \\
\quad+\left.\frac{C_{s} \mu}{\rho T_{0}}\left(\boldsymbol{\nabla} \Theta^{(0)}-\left(\boldsymbol{\nabla} \Theta^{(0)} \cdot \frac{\mathbf{r}}{r}\right) \frac{\mathbf{r}}{r}\right)\right|_{r=a}, \\
\left.\left(\mathbf{v}_{1}-\frac{C_{m} l}{\mu} \mathbf{P}_{\tau 1}\right)\right|_{r=a}=\mathbf{U}_{1}+\mathbf{\Omega}_{1} \times \frac{\mathbf{r}}{r}+\mathbf{B}_{1}+\mathbf{B}_{2}+\mathbf{S}_{1}+\mathbf{S}_{2} \\
+\mathbf{S}_{3},
\end{gathered}
$$

where

$$
\begin{aligned}
\mathbf{B}_{1}= & -\left.a f(\theta, \phi) \frac{\partial}{\partial r}\left(\mathbf{v}_{0}-\frac{C_{m} l}{\mu} \mathbf{P}_{\tau 0}\right)\right|_{r=a}, \\
\mathbf{B}_{2}= & a \frac{C_{m} l}{\mu}\left(-\boldsymbol{\sigma}_{0} \cdot \nabla f+2\left(\frac{\mathbf{r}}{r} \cdot \boldsymbol{\sigma}_{0} \cdot \boldsymbol{\nabla} f\right) \frac{\mathbf{r}}{r}\right. \\
& \left.+\left(\frac{\mathbf{r}}{r} \cdot \boldsymbol{\sigma}_{0} \cdot \frac{\mathbf{r}}{r}\right) \boldsymbol{\nabla} f\right)\left.\right|_{r=a}, \\
\mathbf{S}_{1}= & \left.a f(\theta, \phi) \frac{C_{s} \mu}{\rho T_{0}} \frac{\partial}{\partial r}\left(\boldsymbol{\nabla} \Theta^{(0)}-\left(\boldsymbol{\nabla} \Theta^{(0)} \cdot \frac{\mathbf{r}}{r}\right) \frac{\mathbf{r}}{r}\right)\right|_{r=a}, \\
\mathbf{S}_{2}= & a \frac{C_{s} \mu}{\rho T_{0}}\left(\left(\frac{\mathbf{r}}{r} \cdot\left(\nabla \Theta^{(0)}+\mathbf{G}\right)\right) \boldsymbol{\nabla} f\right. \\
& +\left.\left(\nabla f \cdot\left(\nabla \Theta^{(0)}+\mathbf{G}\right) \frac{\mathbf{r}}{r}\right)\right|_{r=a}, \\
\mathbf{S}_{3}= & \left.\frac{C_{s} \mu}{\rho T_{0}}\left(\boldsymbol{\nabla} \Theta^{(1)}-\left(\boldsymbol{\nabla} \Theta^{(1)} \cdot \frac{\mathbf{r}}{r}\right) \frac{\mathbf{r}}{r}\right)\right|_{r=a},
\end{aligned}
$$

$\boldsymbol{\sigma}_{0}$ and $\mathbf{P}_{\tau 0}$ are the zeroth-order terms of $\boldsymbol{\sigma}$ and $\mathbf{P}_{\tau}$, respectively, and $\mathbf{P}_{\tau 1}$ is the first-order term of $\mathbf{P}_{\tau}$, all being taken on the surface of the unperturbed sphere of radius $a$. Physically, the terms $\mathbf{B}_{1}$ and $\mathbf{S}_{1}$ represent the corrections due to the changes in the size of the particle, $\mathbf{B}_{2}$ and $\mathbf{S}_{2}$ take into account the changes in the shape of the particle, which manifest themselves as local corrections in the direction of the normal vector, while $\mathbf{S}_{3}$ combines both effects of the size and the shape of the particle.

Similarly, expanding boundary condition (9) for the heat flux and condition (10) for the temperature jump at the particle surface, we get

$$
\begin{gathered}
\left.\mathbf{r} \cdot \boldsymbol{\nabla}\left(k_{p} \Psi^{(0)}-k_{f} \Theta^{(0)}\right)\right|_{r=a}=\left(k_{f}-k_{p}\right) a \frac{\mathbf{r}}{r} \cdot \mathbf{G}, \\
\left.\left(\Theta^{(0)}-\Psi^{(0)}-C_{t} l \frac{\mathbf{r}}{r} \cdot \nabla \Theta^{(0)}\right)\right|_{r=a}=C_{t} l \frac{\mathbf{r}}{r} \cdot \mathbf{G}
\end{gathered}
$$

for the zeroth order, and

$$
\begin{aligned}
\mathbf{r} \cdot \boldsymbol{\nabla} & \left.\left(k_{p} \Psi^{(1)}-k_{f} \Theta^{(1)}\right)\right|_{r=a} \\
= & \left.a^{2} f(\theta, \phi) \frac{\partial}{\partial r}\left(\frac{\mathbf{r}}{r} \cdot \nabla\left(k_{f} \Theta^{(0)}-k_{p} \Psi^{(0)}\right)\right)\right|_{r=a} \\
& -\left.a^{2} \boldsymbol{\nabla} f \cdot \boldsymbol{\nabla}\left(k_{f} \Theta^{(0)}-k_{p} \Psi^{(0)}\right)\right|_{r=a} \\
& \quad-\left.\left(k_{f}-k_{p}\right) a^{2} \boldsymbol{\nabla} f \cdot \mathbf{G}\right|_{r=a}, \\
\left.\left(\Theta^{(1)}-\Psi^{(1)}-C_{t} l \frac{\mathbf{r}}{r} \cdot \nabla \Theta^{(1)}\right)\right|_{r=a} & \\
= & \left.C_{t} l\left(a f(\theta, \phi) \frac{\partial}{\partial r}\left(\frac{\mathbf{r}}{r} \cdot \nabla \Theta^{(0)}\right)-a \boldsymbol{\nabla} f \cdot \nabla \Theta^{(0)}\right)\right|_{r=a} \\
& +\left.a f(\theta, \phi) \frac{\partial}{\partial r}\left(\Psi^{(0)}-\Theta^{(0)}\right)\right|_{r=a}-\left.C_{t} l a \boldsymbol{\nabla} f \cdot \mathbf{G}\right|_{r=a}
\end{aligned}
$$

for the first order. Note that the first-order flow field may be determined by satisfying boundary condition (21) at the surface of the unperturbed sphere once the zeroth-order thermal and velocity fields specified by Eqs. (20), (27), and (28) together with the first order thermal field given by Eqs. (29) and (30) have been solved.

\section{SOLUTION OF THE UNPERTURBED PROBLEM}

In this section, the zeroth-order approximation for the temperature and fluid velocity fields around a slightly deformed spherical particle in the small parameter $\varepsilon$ is considered. As it can be shown, ${ }^{33,34}$ to get the solution of Eq. (5) with boundary conditions (14) and (15) for the case of a spherical particle of radius $a$, one has to calculate the following quantities:

$$
\begin{aligned}
& \mathcal{L}_{1}(\mathbf{A})=\left.\frac{1}{r}(\mathbf{r} \cdot \mathbf{A})\right|_{r=a}=\sum_{n} X_{n}(\theta, \phi) \\
& \mathcal{L}_{2}(\mathbf{A})=\left.\left((\mathbf{r} \cdot \boldsymbol{\nabla}) \frac{(\mathbf{r} \cdot \mathbf{A})}{r}-r \boldsymbol{\nabla} \cdot \mathbf{A}\right)\right|_{r=a}=\sum_{n} Y_{n}(\theta, \phi) \\
& \mathcal{L}_{3}(\mathbf{A})=\left.\mathbf{r} \cdot(\boldsymbol{\nabla} \times \mathbf{A})\right|_{r=a}=\sum_{n} Z_{n}(\theta, \phi)
\end{aligned}
$$

where according to Eqs. (20) and (21) $\mathbf{A}=\mathbf{v}-\left(C_{m} l / \mu\right) \mathbf{P}_{\tau}$, while $X_{n}, Y_{n}$ and $Z_{n}$ are surface spherical harmonics. This and similar techniques were widely used for a variety of different boundary conditions, e.g., those describing Stokesian flows around a submerged fluid drop or gas bubble, elastic particle, particle with a slip surface, etc. ${ }^{35-38}$

Applying the expressions for the fluid velocity field in Eq. (6) and tangential stress vector in Eq. (16) to Eqs. (31)-(33), we obtain (only those terms which define the flow in an unbounded fluid, i.e. with negative powers of $r$, are retained)

$\chi_{-n-1}=\frac{1}{n(n+1)\left(1+(n+2) C_{m}^{*}\right)}\left(\frac{a}{r}\right)^{n+1} Z_{n}$, 
$\varphi_{-n-1}=\frac{a}{2(n+1)} \frac{Y_{n}+\left(n+2\left(n^{2}-1\right) C_{m}^{*}\right) X_{n}}{1+(2 n+1) C_{m}^{*}}\left(\frac{a}{r}\right)^{n+1}$,

$p_{-n-1}=\frac{\mu(2 n-1)}{a(n+1)} \frac{Y_{n}+(n+2)\left(1+2 n C_{m}^{*}\right) X_{n}}{1+(2 n+1) C_{m}^{*}}\left(\frac{a}{r}\right)^{n+1}, \quad n \geqslant 1$

where $C_{m}^{*}=C_{m} l / a$. Thus, Eq. (6) and the above equations allow us to describe the structure of the fluid flow when the surface harmonics in Eqs. (31)-(33) are given.

Now we consider the thermal fields inside and outside the particle. As one can see from Eqs. (27)-(30), the boundary conditions can be formulated in the following general way:

$$
\begin{aligned}
& \left.\mathbf{r} \cdot \boldsymbol{\nabla}\left(k^{*} \Psi-\Theta\right)\right|_{r=a}=\sum_{n} L_{n}(\theta, \phi), \\
& \left.\left(\Theta-\Psi-C_{t}^{*} \mathbf{r} \cdot \nabla \Theta\right)\right|_{r=a}=\sum_{n} N_{n}(\theta, \phi),
\end{aligned}
$$

where $k^{*}=k_{p} / k_{f}$ and $C_{t}^{*}=C_{t} l / a$, while $L_{n}(\theta, \phi)$ and $N_{n}(\theta, \phi)$ are surface spherical harmonics. Using Eq. (13) for the boundary conditions (37) and (38), we can write

$$
\begin{aligned}
& \Theta_{-n-1}=\frac{L_{n}+n k^{*} N_{n}}{n+1+n k^{*}\left(1+(n+1) C_{t}^{*}\right)}\left(\frac{r}{a}\right)^{-n-1}, \quad r \geqslant a \\
& \Psi_{n}=\frac{\left(1+(n+1) C_{t}^{*}\right) L_{n}-(n+1) N_{n}}{n+1+n k^{*}\left(1+(n+1) C_{t}^{*}\right)}\left(\frac{r}{a}\right)^{n}, \quad r \leqslant a, \quad n \geqslant 1 .
\end{aligned}
$$

During the derivation of Eqs. (39) and (40), we accounted for the boundary conditions (11) and (12), which require that the temperature field $\Psi$ be finite everywhere in the interior of the particle and the field $\Theta$ vanish at infinity.

Without any loss of generality, in the spherical coordinates with their origin at the center of the undeformed sphere, the perturbation of the surface of the particle defined by Eq. (17) may be expanded into an infinite series of surface spherical harmonics as

$$
f(\theta, \phi)=\sum_{k=0}^{\infty} f_{k}(\theta, \phi) \text {. }
$$

In the following analysis any expression for $\mathbf{B}_{1}^{k}, \mathbf{B}_{2}^{k}, \mathbf{S}_{1}^{k}, \mathbf{S}_{2}^{k}$, and $\mathbf{S}_{3}^{k}$ defined by Eqs. (22)-(26) and $L_{k}$ and $N_{k}$ defined by Eqs. (37), (38), (29), and (30) is assumed to correspond with the particular spherical harmonic $f_{k}(\theta, \phi)$.

As it follows from Newton's second law, when a particle translates with a constant velocity and rotates with a constant angular velocity, the total force and total torque acting on the particle are zero, respectively. This observation allows us to obtain the thermophoretic velocity and angular velocity of the particle. The only force acting on the particle is a hydrodynamic drag force, and the same statement is true with respect to the torque. To evaluate the hydrodynamic drag force and torque acting on a deformed spherical particle, we use the well-known formulas derived in terms of the solid spherical harmonics $p_{-2}$ and $\chi_{-2},{ }^{33,34}$

$$
\mathbf{F}=-4 \pi \boldsymbol{\nabla}\left(r^{3} p_{-2}\right), \quad \mathbf{T}=-8 \pi \mu \boldsymbol{\nabla}\left(r^{3} \chi_{-2}\right) .
$$

The validity of these general expressions for the force and torque are independent of the shape of the particle.

Analogous to Eq. (18), we can expand the force and torque in powers of the perturbation parameter $\varepsilon$,

$$
\mathbf{F}=\sum_{i=0}^{\infty} \varepsilon^{i} \mathbf{F}_{i}, \quad \mathbf{T}=\sum_{i=0}^{\infty} \varepsilon^{i} \mathbf{T}_{i}
$$

Evidently, the thermophoretic velocity and angular velocity of the particle of various orders of $\varepsilon$ are to be determined by the requirement of

$$
\mathbf{F}_{i}=-4 \pi \boldsymbol{\nabla}\left(r^{3} p_{-2}^{(i)}\right)=\mathbf{0}, \quad \mathbf{T}_{i}=-8 \pi \mu \boldsymbol{\nabla}\left(r^{3} \chi_{-2}^{(i)}\right)=\mathbf{0} .
$$

Here, and in the following, the superscript to a solid spherical harmonic indicates the order in the expansion in powers of the small parameter of deformation $\varepsilon$, while the subscript stands for the order of the harmonics.

Now we have everything at hand to find the zeroth-order terms in the expansion of the thermal and hydrodynamic fields. Let us first consider the structure of the temperature distribution. From boundary conditions (27) and (28) and expressions (37) and (38) we readily obtain that the zeroth-order contributing surface spherical harmonics are $L_{1}^{(0)}(\theta, \phi)=a\left(1-k^{*}\right) \mathbf{G} \cdot \mathbf{r} / r$ and $N_{1}^{(0)}(\theta, \phi)=a C_{t}^{*} \mathbf{G} \cdot \mathbf{r} / r$. Thus, using Eqs. (39) and (40), we get the corresponding spherical harmonics

$$
\begin{aligned}
& \Theta_{-2}^{(0)}=H^{*}(\mathbf{G} \cdot \mathbf{r}) \frac{a^{3}}{r^{3}}=\frac{1-k^{*}+k^{*} C_{t}^{*}}{2+k^{*}+2 k^{*} C_{t}^{*}}(\mathbf{G} \cdot \mathbf{r}) \frac{a^{3}}{r^{3}}, \\
& \Psi_{1}^{(0)}=\frac{1-k^{*}-2 k^{*} C_{t}^{*}}{2+k^{*}+2 k^{*} C_{t}^{*}}(\mathbf{G} \cdot \mathbf{r})
\end{aligned}
$$

which reproduces the result for the temperature field obtained previously. ${ }^{32}$

When the solution for the zeroth-order thermal field is obtained, we continue with the velocity field. Taking into account boundary condition (20) and performing elementary algebraic calculations, we see that, among the surface harmonics in Eqs. (31)-(33), the following zeroth-order ones are nonzero:

$$
\begin{aligned}
X_{1}^{(0)}(\theta, \phi) & =\frac{\mathbf{U}_{0} \cdot \mathbf{r}}{r}=U_{0} \cos \theta, \\
Y_{1}^{(0)}(\theta, \phi) & =\left.\frac{2 C_{s} \mu}{a \rho T_{0}}\left(\Theta^{(0)}+\mathbf{G} \cdot \mathbf{r}\right)\right|_{r=a} \\
& =\frac{2 C_{s} \mu}{\rho T_{0}} \frac{\mathbf{G} \cdot \mathbf{r}}{r}\left(H^{*}+1\right), \\
Z_{1}^{(0)}(\theta, \phi) & =\left.2 \mathbf{\Omega}_{0} \cdot \mathbf{r}\right|_{r=a}=2 a \Omega_{0} \cos \theta,
\end{aligned}
$$

where $H^{*}$ is defined by Eq. (45). Applying Eqs. (34)-(36) to the above equations and substituting the result into Eqs. (44) with $i=0$ lead to the following expressions for the thermophoretic drift velocity and angular velocity 


$$
\begin{aligned}
& \mathbf{U}_{0}=-M^{*} \mathbf{G}=-\frac{2 C_{s} \mu\left(1+k^{*} C_{t}^{*}\right)}{\rho T_{0}\left(1+2 C_{m}^{*}\right)\left(2+k^{*}+2 k^{*} C_{t}^{*}\right)} \mathbf{G}, \\
& \mathbf{\Omega}_{0}=\mathbf{0} .
\end{aligned}
$$

The latter result follows immediately from the isotropy of the zeroth-order problem. As we see, Eq. (49) reproduces the result given by Eqs. (1) and (4).

From Eqs. (34)-(36) and (46)-(50), we observe that

$$
\varphi_{-2}^{(0)}=-\frac{a^{3}}{2 r^{3}}\left(\mathbf{U}_{0} \cdot \mathbf{r}\right), \quad p_{-2}^{(0)}=0, \quad \chi_{-2}^{(0)}=0 .
$$

Recalling the general expression (6) for the velocity field, we get from Eq. (51) the structure of the flow

$$
\mathbf{v}_{0}=\boldsymbol{\nabla} \varphi_{-2}^{(0)}=-\frac{a^{3}}{2 r^{3}} \mathbf{U}_{0}+\frac{3 a^{3}}{2 r^{3}} \frac{\left(\mathbf{U}_{0} \cdot \mathbf{r}\right) \mathbf{r}}{r^{2}} .
$$

As one can easily see, the above expression reproduces the result for velocity field obtained previously. ${ }^{32}$

\section{SOLUTION OF THE THERMAL AND HYDRODYNAMIC FIELDS OF THE PERTURBED PROBLEM}

Within this section, the scalars $L_{n}^{(1)}$ and $N_{n}^{(1)}$ defined by Eqs. (37), (38), (29), and (30) and the vectors $\mathbf{B}_{1}, \mathbf{B}_{2}, \mathbf{S}_{1}, \mathbf{S}_{2}$, and $\mathbf{S}_{3}$ in Eqs. (21)-(26) are treated. Following Brenner's approach, ${ }^{33}$ we define surface spherical harmonics

$$
\begin{aligned}
& W_{k-1}=r^{-k+1} \mathbf{G} \cdot \boldsymbol{\nabla}\left(r^{k} f_{k}\right)=r \mathbf{G} \cdot \boldsymbol{\nabla} f_{k}+k \frac{\mathbf{G} \cdot \mathbf{r}}{r} f_{k}, \\
& V_{k+1}=r^{k+2} \mathbf{G} \cdot \boldsymbol{\nabla}\left(r^{-k-1} f_{k}\right)=r \mathbf{G} \cdot \boldsymbol{\nabla} f_{k}-(k+1) \frac{\mathbf{G} \cdot \mathbf{r}}{r} f_{k}, \\
& M_{k}=\mathbf{G} \cdot\left(\boldsymbol{\nabla} \times\left(\mathbf{r} f_{k}\right)\right)
\end{aligned}
$$

with respect to the surface spherical harmonics $f_{k}$.

Substituting Eq. (45) for the zeroth-order thermal fields into Eqs. (29) and (30), for the surface spherical harmonics $L_{n}^{(1)}$ and $N_{n}^{(1)}$ we get

$L_{k-1}^{(1)}=-3 a H^{*} \frac{k-1}{2 k+1} W_{k-1}$,

$L_{k+1}^{(1)}=-3 a H^{*} \frac{k+2}{2 k+1} V_{k+1}$,

$N_{k-1}^{(1)}=-3 a \frac{-1+k^{*}+(k-1) k^{*} C_{t}^{* 2}+\left(k-1+2 k^{*}\right) C_{t}^{*}}{(2 k+1)\left(2+k^{*}+2 k^{*} C_{t}^{*}\right)} W_{k-1}$,

$N_{k+1}^{(1)}=-3 a \frac{1-k^{*}+(k+2) k^{*} C_{t}^{* 2}+\left(k+2-2 k^{*}\right) C_{t}^{*}}{(2 k+1)\left(2+k^{*}+2 k^{*} C_{t}^{*}\right)} V_{k+1}$,

where $L_{k-1}^{(1)}, L_{k+1}^{(1)}, N_{k-1}^{(1)}$, and $N_{k+1}^{(1)}$ are the only nonzero contributions due to any particular $f_{k}$. The above expressions to- gether with Eqs. (39)-(41) allow one to construct the solution for the first-order thermal field.

Substituting expression (52) for the zeroth-order velocity field into expression (22) for $\mathbf{B}_{1}$ and applying the procedure defined by Eqs. (31)-(33), we obtain

$$
\mathcal{L}_{1}\left(\mathbf{B}_{1}^{k}\right)=\frac{3 M^{*}}{2 k+1}\left(V_{k+1}-W_{k-1}\right)
$$

$$
\begin{aligned}
\mathcal{L}_{2}\left(\mathbf{B}_{1}^{k}\right)= & -\frac{3 M^{*}\left(k-5+8(k-1) C_{m}^{*}\right)}{2(2 k+1)} W_{k-1} \\
& -\frac{3 M^{*}\left(k+6+8(k+2) C_{m}^{*}\right)}{2(2 k+1)} V_{k+1},
\end{aligned}
$$

$$
\mathcal{L}_{3}\left(\mathbf{B}_{1}^{k}\right)=-\frac{3 M^{*}\left(1+8 C_{m}^{*}\right)}{2} M_{k}
$$

where $M^{*}$ is defined by Eq. (49). This furnishes the expansion of $\mathbf{B}_{1}$ in the sum of spherical harmonics.

For the term $\mathbf{B}_{2}$ defined by Eq. (23), we obtain

$$
\mathcal{L}_{1}\left(\mathbf{B}_{2}^{k}\right)=-\frac{3 M^{*} C_{m}^{*}}{2 k+1}\left(k V_{k+1}+(k+1) W_{k-1}\right)
$$

$$
\mathcal{L}_{2}\left(\mathbf{B}_{2}^{k}\right)=\frac{3 M^{*} C_{m}^{*}}{2 k+1}\left((k+1)(3 k-1) W_{k-1}-k(3 k+4) V_{k+1}\right),
$$

$$
\mathcal{L}_{3}\left(\mathbf{B}_{2}^{k}\right)=9 M^{*} C_{m}^{*} M_{k}
$$

Again, these three equations furnish the expansion of $\mathbf{B}_{2}$ in surface spherical harmonics.

For the term $\mathbf{S}_{1}$ given as in Eq. (24), we get

$$
\begin{aligned}
& \mathcal{L}_{1}\left(\mathbf{S}_{1}^{k}\right)=0 \\
& \mathcal{L}_{2}\left(\mathbf{S}_{1}^{k}\right)=\frac{3 C_{s} \mu}{\rho T_{0}} \frac{H^{*}}{2 k+1}\left((k-1) W_{k-1}+(k+2) V_{k+1}\right), \\
& \mathcal{L}_{3}\left(\mathbf{S}_{1}^{k}\right)=\frac{3 C_{s} \mu}{\rho T_{0}} H^{*} M_{k} .
\end{aligned}
$$

This furnishes the expansion of $\mathbf{S}_{1}$ in the sum of spherical harmonics.

Similarly, for $\mathbf{S}_{2}$ as in Eq. (25) we obtain 


$$
\begin{aligned}
\mathcal{L}_{1}\left(\mathbf{S}_{2}^{k}\right)= & \frac{C_{s} \mu}{\rho T_{0}} \frac{H^{*}+1}{2 k+1}\left((k+1) W_{k-1}+k V_{k+1}\right), \\
\mathcal{L}_{2}\left(\mathbf{S}_{2}^{k}\right)= & -\frac{3 C_{s} \mu(k+1)\left(2-(k-1) k^{*}+2 k^{*} C_{t}^{*}\right)}{\rho T_{0}(2 k+1)\left(2+k^{*}+2 k^{*} C_{t}^{*}\right)} W_{k-1} \\
& -\frac{3 C_{s} \mu k\left(2+2 k^{*}+k k^{*}+2 k^{*} C_{t}^{*}\right)}{\rho T_{0}(2 k+1)\left(2+k^{*}+2 k^{*} C_{t}^{*}\right)} V_{k+1}, \\
\mathcal{L}_{3}\left(\mathbf{S}_{2}^{k}\right)= & \frac{C_{s} \mu}{\rho T_{0}}\left(1-2 H^{*}\right) M_{k} .
\end{aligned}
$$

This furnishes the expansion of $\mathbf{S}_{2}$ in the sum of spherical harmonics.
To obtain expressions (31)-(33) for $\mathbf{S}_{3}$ defined by Eq. (26), we note that, for a solid spherical harmonic $\Theta_{m}^{(1)}$ of an arbitrary order $m$, the following relations are valid:

$$
\begin{aligned}
& \mathcal{L}_{1}\left(\mathbf{S}_{3}^{m}\right)=0, \\
& \mathcal{L}_{2}\left(\mathbf{S}_{3}^{m}\right)=\left.\frac{C_{s} \mu}{\rho T_{0}} \frac{m(m+1)}{a} \Theta_{m}^{(1)}\right|_{r=a}, \\
& \mathcal{L}_{3}\left(\mathbf{S}_{3}^{m}\right)=0 .
\end{aligned}
$$

Now, using the surface spherical harmonics given by Eqs. (56)-(59) by means of Eq. (39), we construct the explicit expression for the $\Theta_{m}^{(1)}$ and thus obtain the closed form of Eq. (73),

$$
\begin{aligned}
\mathcal{L}_{2}\left(\mathbf{S}_{3}^{k}\right)= & \frac{C_{s} \mu}{\rho T_{0}} \frac{3 k(k-1)^{2}\left(1-k^{*}\left(2-k C_{t}^{*}\right)+k^{* 2}\left(1+2 C_{t}^{*}+(k-1) C_{t}^{* 2}\right)\right)}{(2 k+1)\left(2+k^{*}+2 k^{*} C_{t}^{*}\right)\left(k^{*}-k\left(1+k^{*}+(k-1) k^{*} C_{t}^{*}\right)\right)} W_{k-1} \\
& -\frac{C_{s} \mu}{\rho T_{0}} \frac{3(k+1)(k+2)}{(2 k+1)\left(2+k^{*}+2 k^{*} C_{t}^{*}\right)}\left(\frac{(k+2)\left(1-k^{*}\left(1-C_{t}^{*}\right)\right)}{k+2+(k+1) k^{*}\left(1+(k+2) C_{t}^{*}\right)}\right. \\
& \left.+\frac{(k+1) k^{*}\left(1-k^{*}+\left(k+2-2 k^{*}\right) C_{t}^{*}+(k+2) k^{*} C_{t}^{* 2}\right)}{k+2+(k+1) k^{*}\left(1+(k+2) C_{t}^{*}\right)}\right) V_{k+1} .
\end{aligned}
$$

Finally, having expressed the vectors $\mathbf{B}_{1}, \mathbf{B}_{2}, \mathbf{S}_{1}, \mathbf{S}_{2}$, and $\mathbf{S}_{3}$ in Eq. (21) in terms of surface spherical harmonics as shown in Eqs. (60)-(75), and utilizing the relations (31)-(36), one can construct the first-order corrections to the background temperature and velocity fields given by Eqs. (45) and (52) as infinite sums over the angular terms $f_{k}(\theta, \phi)$ in Eq. (41) relevant to a particular perturbation of the shape of a spherical particle. Note that, in order to obtain physically important characteristics of the motion of the particle, such as the thermophoretic drift and rotational velocities, all sufficient information can be extracted from the finite number of terms in the infinite sums of Eq. (6) representing the velocity field. In the upcoming sections, we will obtain the explicit translational and angular velocities of the particle, consider their general features, and address some important special cases, such as prolate and oblate spheroidal particles.

\section{THERMOPHORETIC VELOCITY OF A DEFORMED SPHERICAL PARTICLE}

Following the procedure explained in Sec. IV and extracting appropriate terms from the expressions given in the previous section, we get

$$
\begin{aligned}
p_{-2}^{(1)}= & \frac{3 a \mu}{2 r^{3}} \frac{1+2 C_{m}^{*}}{1+3 C_{m}^{*}}\left(\frac{C_{s} \mu}{\rho T_{0}}\left(2 D_{1}(\mathbf{G} \cdot \mathbf{r}) f_{0}+D_{2} \mathbf{G} \cdot \nabla\left(r^{2} f_{2}\right)\right)\right. \\
& \left.+\left(\mathbf{U}_{1} \cdot \mathbf{r}\right)\right) \\
\chi_{-2}^{(1)}= & \frac{a^{2}}{r^{2}} \frac{1}{1+3 C_{m}^{*}}\left(\mathbf{\Omega}_{1} \cdot \mathbf{r}\right),
\end{aligned}
$$

where

$$
\begin{aligned}
& D_{1}=\frac{8 k^{*} C_{t}^{*} C_{m}^{*}+4 k^{* 2} C_{t}^{* 2} C_{m}^{*}+2\left(k^{*}+2\right) C_{m}^{*}-k^{* 2} C_{t}^{*}}{\left(1+2 C_{m}^{*}\right)^{2}\left(2+k^{*}+2 k^{*} C_{t}^{*}\right)^{2}}, \\
& D_{2}=\frac{k^{*}\left(9+C_{t}^{*} k^{*}\right)+2 C_{m}^{*}\left(16+k^{*}\left(17+32 C_{t}^{*}\right)+k^{* 2} C_{t}^{*}\left(9+16 C_{t}^{*}\right)\right)}{5\left(1+2 C_{m}^{*}\right)^{2}\left(2+k^{*}+2 k^{*} C_{t}^{*}\right)^{2}} .
\end{aligned}
$$


Equations (76) and (77) are consistent with the observation that only the terms $f_{n}$ and $f_{n-2}$ contribute to the pressure harmonics $p_{-n}^{(1)}$ and only the term $f_{n-1}$ may contribute to the vortical harmonics $\chi_{-n}^{(1)}$ for the translational motion of a slightly deformed sphere. ${ }^{33,34,38}$

The substitution of (76) and (77) into Eq. (44) taking $i$ $=1$ allows us to write the first-order drift velocity and angular velocity of the arbitrary, slightly deformed spherical particle,

$$
\begin{aligned}
& \mathbf{U}_{1}=-\frac{C_{s} \mu}{\rho T_{0}} \boldsymbol{\Phi}^{(1)} \cdot \mathbf{G}, \\
& \Omega_{1}=\mathbf{0}
\end{aligned}
$$

where the thermophoretic mobility tensor

$$
\Phi_{i j}^{(1)}=2 D_{1} f_{0} \delta_{i j}+D_{2} \nabla_{i} \nabla_{j}\left(r^{2} f_{2}\right),
$$

where $\delta_{i j}$ is the Kronecker delta and components of the tensor are given in a Cartesian frame. As we can see, this tensor is symmetric and depends solely upon the geometrical properties of the particle surface $\left[f_{0}\right.$ is a constant and $\nabla_{i} \nabla_{j}\left(r^{2} f_{2}\right)$ is a constant symmetric dyadic for a specified particle shape]. We note that the first-order correction to the thermophoretic drift velocity is entirely defined by the zeroth- and secondorder terms in the angular perturbation of the particle shape. The outcome that only the terms $f_{0}$ and $f_{2}$ contribute to the leading-order correction to the particle velocity may be traced directly to the fact that the Stokes equations (5) satisfy the biharmonic vector equation $\nabla^{4} \mathbf{v}=0 .{ }^{33}$

Concerning the fact that the angular velocity is zero irrespective of the shape of the particle, we note that a similar situation occurs in the case of thin electrical-double-layer electrophoretic motion, ${ }^{39,40}$ in which there is no frictional slip or potential jump at the particle surface, the particle velocity is independent of the particle size, and the fluid flow around the charged particle is purely irrotational. Thus, our result in Eq. (81) is the first indication that the thermophoretic flow in the case of finite Knudsen number is also irrotational for a particle of an arbitrary shape and with a temperature jump and a frictional slip at its surface.

The thermophoretic velocity of a spherical particle is given by Eq. (49). If the radius of a spherical particle acquires a small uniform increment $a \varepsilon f_{0}$, then the thermophoretic velocity changes accordingly [up to $O(\varepsilon)$, we are reminded here that both $C_{m}^{*}$ and $C_{t}^{*}$ depend on the radius $a$ of the particle]

$$
\mathbf{U}_{0}\left(a\left(1+\varepsilon f_{0}\right)\right)-\mathbf{U}_{0}(a)=a f_{0} \varepsilon \frac{\partial}{\partial a} \mathbf{U}_{0}(a)=-\varepsilon f_{0} \frac{C_{s} \mu}{\rho T_{0}} 2 D_{1} \mathbf{G}
$$

which coincides with the appropriate term in Eq. (82). This observation reveals that the origin of the $f_{0}$ term in the thermophoretic velocity is entirely dictated by the change in the size of the particle, but not by its shape.

\section{THERMOPHORETIC VELOCITY OF A SPHEROID}

As particular case of a slightly deformed sphere, we consider the following form of a spheroidal particle in the Cartesian frame $(x, y, z)$,

$$
\frac{x^{2}+y^{2}}{a^{2}}+\frac{z^{2}}{a^{2}(1-\varepsilon)^{2}}=1 .
$$

For the case $\varepsilon>0$ the spheroid is oblate; for the case $\varepsilon<0$ the spheroid is prolate. To $O(\varepsilon)$, the shape of the particle can be expressed by Eqs. (17) and (41) with only two nonzero $f_{k}$ 's,

$$
f_{0}=-\frac{1}{3}, \quad f_{2}=\frac{1}{3}-\cos ^{2} \theta .
$$

Using the above formulas and Eq. (82) for the first-order thermophoretic mobility tensor $\widetilde{\boldsymbol{\Phi}}^{(1)}$ in the spheroidal geometry, we get

$$
\begin{aligned}
\widetilde{\Phi}_{i j}^{(1)}= & -\frac{2\left[k^{*}\left(3+2 k^{*} C_{t}^{*}\right)+2 C_{m}^{*}\left(2+4 k^{*}\left(1+C_{t}^{*}\right)+k^{* 2} C_{t}^{*}\left(3+2 C_{t}^{*}\right)\right)\right]}{5\left(1+2 C_{m}^{*}\right)^{2}\left(2+k^{*}+2 k^{*} C_{t}^{*}\right)^{2}} \delta_{i j} \\
& +\frac{2\left[k^{*}\left(9+k^{*} C_{t}^{*}\right)+2 C_{m}^{*}\left(16+k^{*}\left(17+32 C_{t}^{*}\right)+k^{* 2} C_{t}^{*}\left(9+16 C_{t}^{*}\right)\right)\right]}{5\left(1+2 C_{m}^{*}\right)^{2}\left(2+k^{*}+2 k^{*} C_{t}^{*}\right)^{2}} \delta_{i 3} \delta_{j 3} .
\end{aligned}
$$

Concerning the axisymmetric motion of a spheroid, i.e., $\mathbf{G}=G_{l} \mathbf{e}_{z}$ and $\mathbf{U}_{1}=U_{1 l} \mathbf{e}_{z}$ in which $\mathbf{e}_{z}$ is the unit vector in the $z$ direction, using Eq. (86) we have the simple compact expression for the drift velocity of the first order,

$$
U_{1 l}=\frac{C_{s} \mu}{\rho T_{0}} \frac{2\left[k^{*}\left(6-k^{*} C_{t}^{*}\right)+2 C_{m}^{*}\left(14+k^{*}\left(13+28 C_{t}^{*}\right)+2 k^{* 2} C_{t}^{*}\left(3+7 C_{t}^{*}\right)\right)\right]}{5\left(1+2 C_{m}^{*}\right)^{2}\left(2+k^{*}+2 k^{*} C_{t}^{*}\right)^{2}} G_{l} .
$$

Similarly, in the case when the external temperature gradient is perpendicular to the axis of revolution of the spheroid, i.e., $\mathbf{G} \cdot \mathbf{e}_{z}=0, \mathbf{U}_{1} \cdot \mathbf{e}_{z}=0,|\mathbf{G}|=G_{t}$, and $\left|\mathbf{U}_{1}\right|=U_{1 t}$, we obtain 


$$
U_{1 t}=-\frac{C_{s} \mu}{\rho T_{0}} \frac{2\left[k^{*}\left(3+2 k^{*} C_{t}^{*}\right)+2 C_{m}^{*}\left(2+4 k^{*}\left(1+C_{t}^{*}\right)+k^{* 2} C_{t}^{*}\left(3+2 C_{t}^{*}\right)\right)\right]}{5\left(1+2 C_{m}^{*}\right)^{2}\left(2+k^{*}+2 k^{*} C_{t}^{*}\right)^{2}} G_{t} .
$$

When the frictional slip and temperature jump do not occur on the surface of the particle (i.e., $C_{m}^{*}=0$ and $C_{t}^{*}=0$ ) the problem of the thermophoresis of a spheroidal particle in a uniform temperature gradient is solvable in the spheroidal coordinate systems by a method of separation of variables. Recently, Keh and $\mathrm{Ou}^{30}$ derived an exact expression for the thermophoretic drift velocity of a spheroidal particle in the case when the external uniform temperature gradient is oriented arbitrarily relative to the axis of symmetry of the particle, which has the form

$$
\begin{aligned}
& U_{l}=-\frac{C_{s} \mu}{\rho T_{0}} G_{l}\left(1+\frac{\alpha}{\beta} k^{*}\right)^{-1}, \\
& U_{t}=-\frac{C_{s} \mu}{\rho T_{0}} G_{t}\left(1+\frac{\beta}{\alpha+1} k^{*}\right)^{-1}
\end{aligned}
$$

for the longitudinal and transversal components, respectively, with
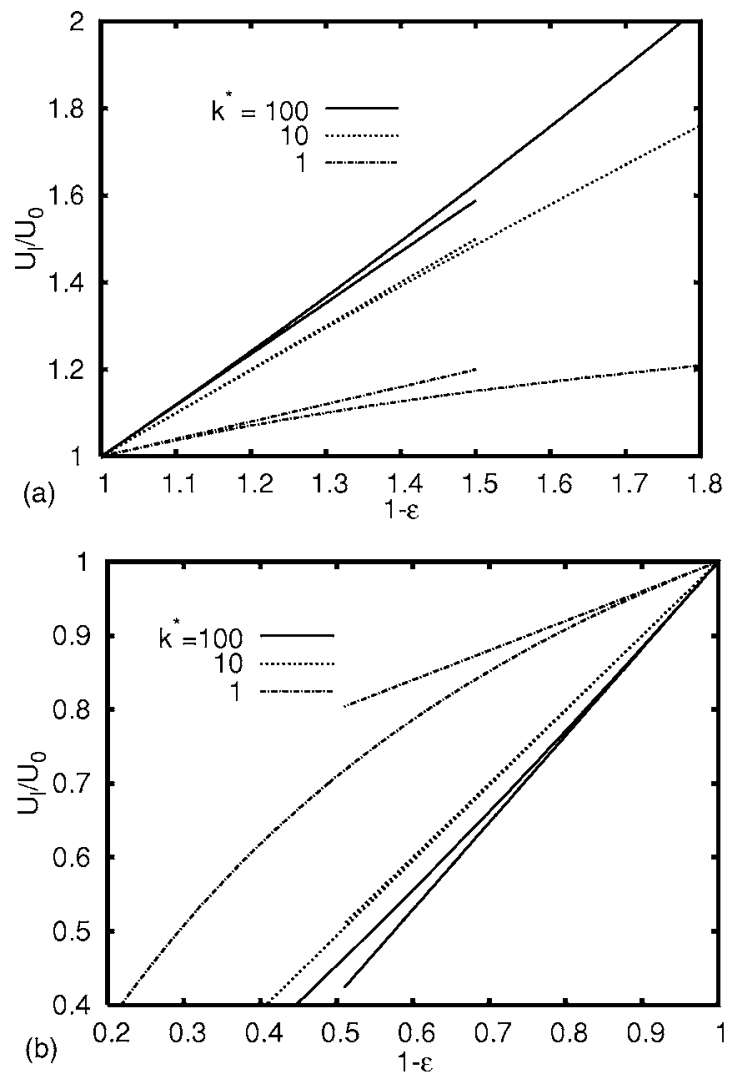

$$
\begin{aligned}
& \alpha=\left(\lambda_{0}^{2}-1\right)\left(\lambda_{0} \operatorname{coth}^{-1} \lambda_{0}-1\right), \\
& \beta=\lambda_{0}\left(\lambda_{0}-\left(\lambda_{0}^{2}-1\right) \operatorname{coth}^{-1} \lambda_{0}\right), \\
& \lambda_{0}=\left[1-(1-\varepsilon)^{-2}\right]^{-1 / 2} .
\end{aligned}
$$

Substituting $C_{t}^{*}=0$ and $C_{m}^{*}=0$ in Eqs. (87) and (88), we get

$$
U_{1 l}=\frac{C_{s} \mu}{\rho T_{0}} \frac{12 k^{*}}{5\left(2+k^{*}\right)^{2}} G_{l}, \quad U_{1 t}=-\frac{C_{s} \mu}{\rho T_{0}} \frac{6 k^{*}}{5\left(2+k^{*}\right)^{2}} G_{t} .
$$

These expressions reproduce the appropriate terms in the expansions of Eq. (89) in powers of the parameter $\varepsilon$.

We proceed with the discussion of the obtained results. Figure 1 displays a comparison of the spheroid velocity between Eq. (89) and Eq. (92) normalized by the corresponding sphere velocity given by Eq. (3). It can be seen that the approximate solution for the thermophoretic velocity to the first order in $\varepsilon$ for the case of zero thermal jump and fric-
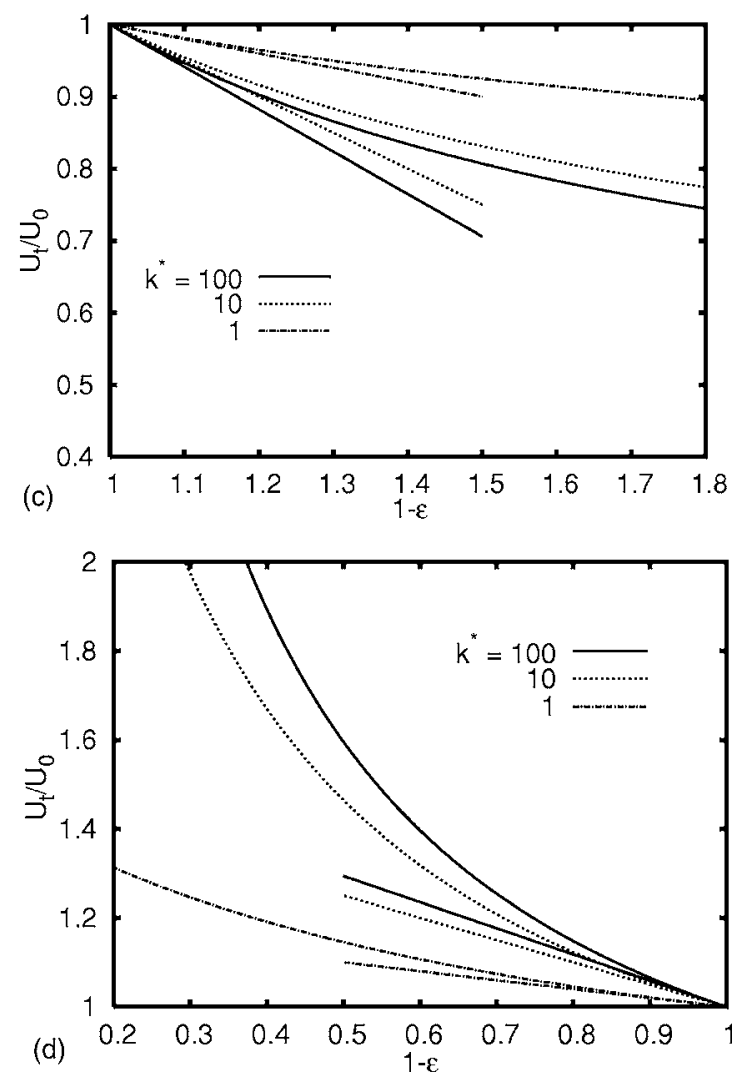

FIG. 1. The normalized thermophoretic velocity for the longitudinal (left) and transversal (right) motions of prolate (upper row) and oblate (lower row) spheroids as functions of the aspect ratio for various values of the thermal conductivity parameter $k^{*}$ and zero thermal jump and frictional slip $\left(C_{t}^{*}=0, C_{m}^{*}\right.$ $=0$ ). The short curves represent the approximation given by Eq. (92), while the long curves denote the exact solution given by Eq. (89). 

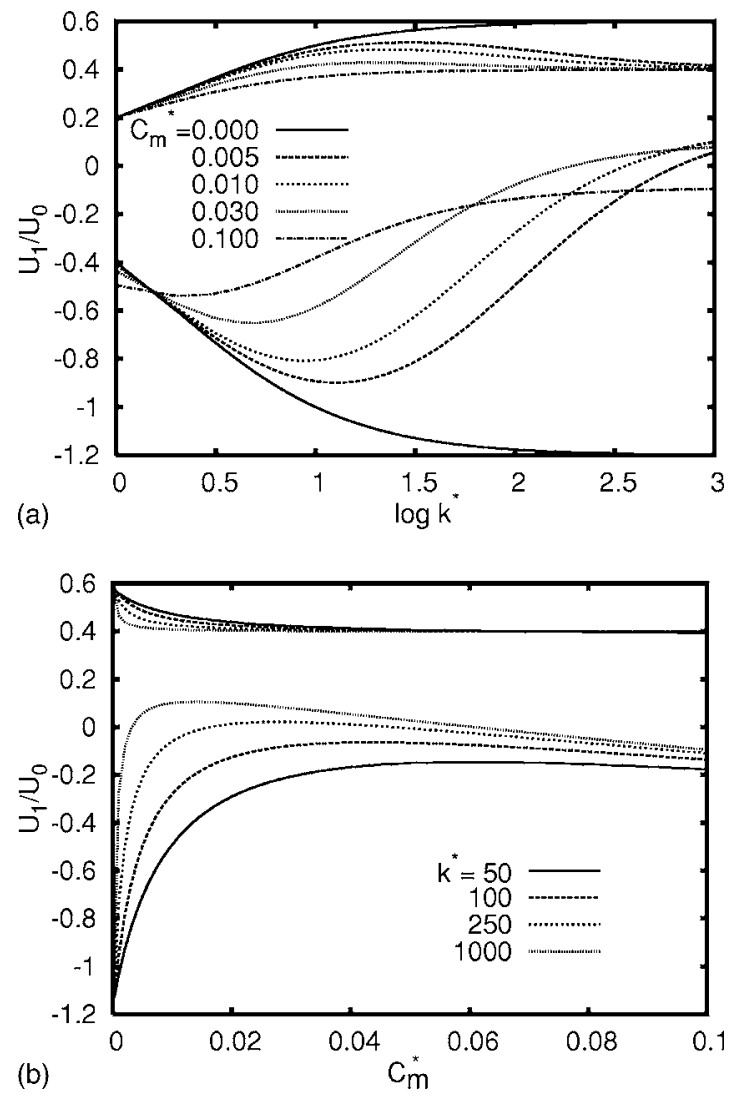

FIG. 2. The normalized first-order coefficient for the expansion of the drift velocity of a spheroid undergoing the longitudinal (lower curves) and transversal (upper curves) thermophoresis given by Eqs. (87) and (88) as functions of the thermal conductivity parameter $k^{*}$ and of the frictional slip coefficient $C_{m}^{*}$ with $C_{t}^{*}=2 C_{m}^{*}$.

tional slip closely follows the exact one up to the values of the aspect ratio $1-\varepsilon$ equal to about 0.5 in the oblate case and about 1.5 in the prolate case for the longitudinal motion of a spheroid and about 0.7 and 1.3 , respectively, for the transversal motion. Thus, we establish the interval of aspect ratio values where it is safe to implement the approximation given by Eqs. (87) and (88). Note that, when the value of the thermal conductivity ratio $k^{*}$ is in its practical range $\left(k^{*}>1\right)$, the approximation deviates faster from the exact solution in the case of transversal motion of a spheroid.

The behavior of the first-order coefficient in the expansion of the thermophoretic drift velocity of a spheroid with respect to the parameter $\varepsilon$ given by Eqs. (87) and (88) as functions of $k^{*}$ and $C_{m}^{*}$ is shown in Fig. 2 (with the common assumption $C_{t}^{*}=2 C_{m}^{*}$ about the values of the frictional slip and thermal jump coefficients). Now, the zeroth-order particle velocity given by Eq. (49) taking the same characteristics is used to normalize the velocity coefficient. One can see that, depending on the values of $k^{*}$ and $C_{m}^{*}$, the normalized first-order coefficient for the longitudinal drift velocity can be either negative or positive (shown as lower curves in Fig. 2), although in the transversal case the first-order velocity correction does not change sign (upper curves in Fig. 2). Both longitudinal and transversal first-order velocity correction terms demonstrate nonmonotonic behavior as a function of the thermal conductivity parameter $k^{*}$. When considered as a function of the frictional slip parameter $C_{m}^{*}$, the longitudinal thermophoretic velocity correction demonstrates nonmonotonic behavior, and the velocity changes sign for the values of the parameter $k^{*}$ greater than about 250, while the transversal velocity correction is always positive and monotonically decreases with an increase in $C_{m}^{*}$ for any given value of $k^{*}$. Since the effects of the three parameters $C_{t}^{*}, C_{m}^{*}$, and $k^{*}$ on the first-order thermophoretic velocity given by Eqs. (87) and (88) interact with one another in quite a complicated manner, it would be very difficult to provide some physical analysis for the above observations from Fig. 2.

The numerical results of the thermophoretic velocity given by Eqs. (87) and (88) for a spheroid as functions of the aspect ratio for various values of the thermal conductivity ratio $k^{*}$ and frictional slip parameter $C_{m}^{*}$ are plotted in Figs. 3 and 4, respectively. Again, the magnitudes of this velocity are normalized by the corresponding value of a spherical particle given by Eq. (49). The cases of $k^{*}<1$, which is not likely to exist in practice, is considered here for the sake of numerical comparison. For a spheroid with a given aspect ratio, Figs. 3 and 4 indicate that the normalized thermophoretic mobility is not necessarily a monotonic function of $k^{*}$ or $C_{m}^{*}$, keeping the other as a constant, which is consistent with the trend shown in Fig. 2.

Equations (87) and (88) provide the longitudinal and transversal components, respectively, of the thermophoretic mobility of an isolated spheroid. In practical applications of thermophoresis, however, collections of particles are encountered. It can be shown that, for an ensemble of identical, noninteracting spheroids with random orientation distribution, the average thermophoretic mobility correct to the first order of $\varepsilon$ can be calculated from these two equations as the sum of one third of $U_{l} / G_{l}$ and two thirds of $U_{t} / G_{t}{ }^{30}$

\section{CONCLUSIONS}

In this paper, we have generalized the perturbation technique developed by Brenner ${ }^{33}$ for the fluid flow past a slightly deformed no-slip sphere to the case of thermophoresis of a slip particle with a temperature jump at its surface and calculated the first-order correction to the temperature and flow fields due to the slight but arbitrary perturbation of the spherical surface of the particle. This analysis is furnished for the case of a surrounding uniform thermal gradient. We derive the closed-form expressions for the thermophoretic drift and angular velocities, and demonstrate that the motion of the particle is irrotational irrespective of its shape. Finally, some cases of the particle of special interest, i.e., prolate and oblate spheroids oriented arbitrarily relative to the imposed temperature gradient, are studied. A comparison of our first-order approximation with the available exact solution shows that the agreement between the two solutions is quite good, even for relatively large deformations of the spherical shape of the particle such as $|\varepsilon|=0.3$. The thermophoretic mobility of a spheroid is found to be a sensitive function of the relative physical and surface properties, the aspect ratio, and the relative orientation of the particle. 

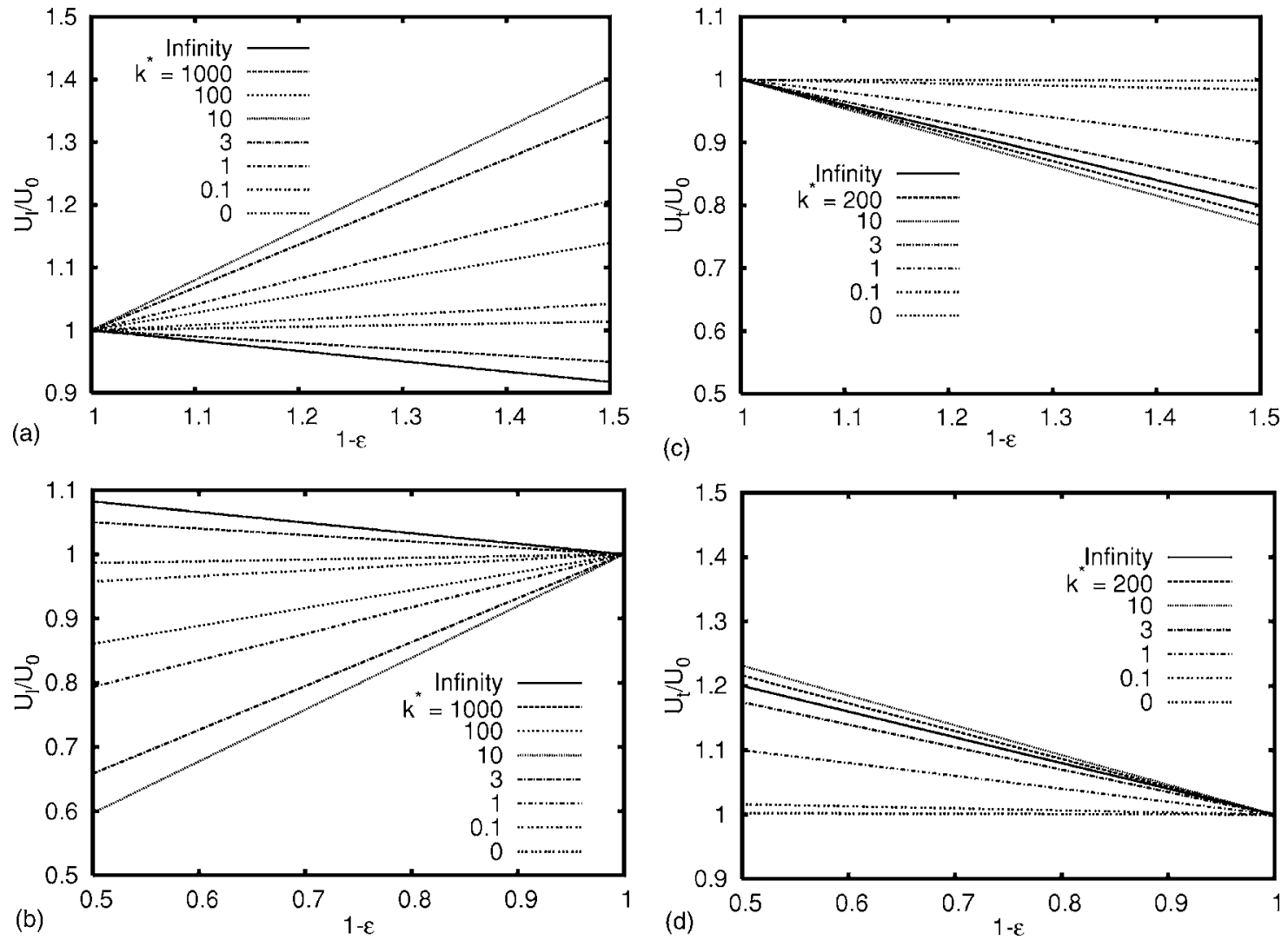

FIG. 3. The normalized thermophoretic velocity for the longitudinal (left) and transversal (right) motions of prolate (upper row) and oblate (lower row) spheroids as functions of the aspect ratio for various values of the thermal conductivity parameter $k^{*}$ and fixed values of the temperature jump and frictional slip parameters, $C_{t}^{*}=2 C_{m}^{*}=0.02$.
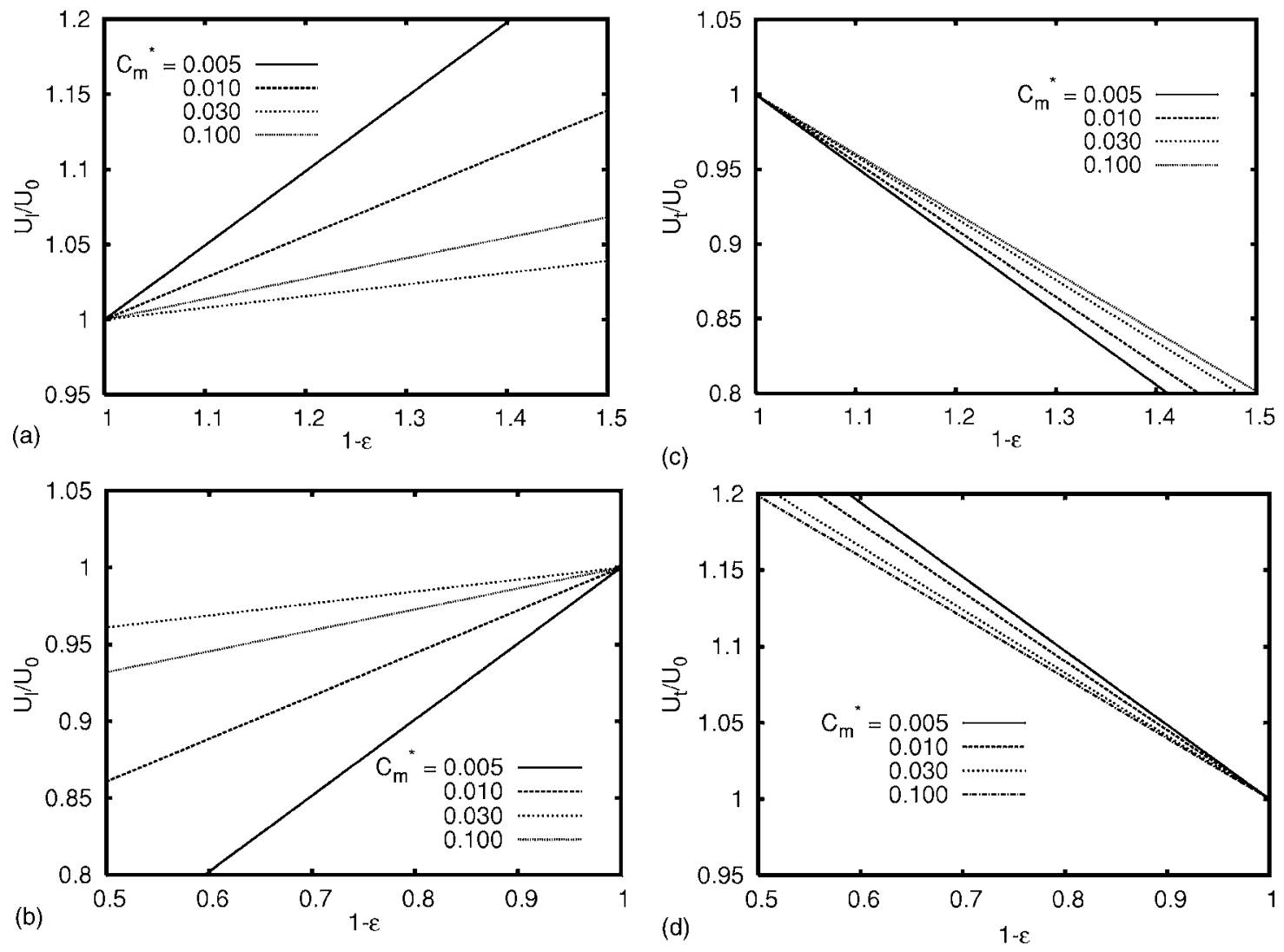

FIG. 4. The normalized thermophoretic velocity for the longitudinal (left) and transversal (right) motions of prolate (upper row) and oblate (lower row) spheroids as functions of the aspect ratio for various values of the frictional slip parameter $C_{m}^{*}=C_{t}^{*} / 2$ and a fixed value of the thermal conductivity parameter $k^{*}=100$. 


\section{ACKNOWLEDGMENT}

This research project was supported by the National Science Council of the Republic of China, under Grant No. NSC 94-2811-002-024.

${ }^{1}$ L. Waldmann and K. H. Schmitt, "Thermophoresis and diffusiophoresis of aerosols," Aerosol Science, edited by C. N. Davies (Academic, New York, 1966).

${ }^{2}$ E. H. Kennard, Kinetic Theory of Gases (McGraw-Hill, New York, 1938).

${ }^{3}$ S. K. Friedlander, Smoke, Dust and Haze (Wiley, New York, 1977).

${ }^{4}$ G. K. Batchelor and C. Shen, "Thermophoretic deposition of particles in gas flowing over cold surfaces," J. Colloid Interface Sci. 107, 21 (1985).

${ }^{5} \mathrm{~N}$. Montassier, D. Boulaud, and A. Renoux, "Experimental study of thermophoretic particle deposition in laminar tube flow," J. Aerosol Sci. 22, 677 (1991).

${ }^{6} \mathrm{M}$. C. Weinberg, "Thermophoretic efficiency in modified chemical vapor deposition process," J. Am. Ceram. Soc. 65, 81 (1982).

${ }^{7}$ N. P. Balsara, R. S. Subramanian, "The influence of buoyancy on thermophoretic deposition of aerosol particles in a horizontal tube," J. Colloid Interface Sci. 118, 3 (1987).

${ }^{8}$ Y. Ye, D. Y. H. Pui, B. Y. H. Liu, S. Opiolka, S. Blumhorst, and H. Fissan, "Thermophoretic effect of particle deposition on a free standing semiconductor wafer in a clean room," J. Aerosol Sci. 22, 63 (1991).

${ }^{9}$ M. M. R. Williams and S. K. Loyalka, Aerosol Science: Theory and Practice, with Special Applications to the Nuclear Industry (Pergamon, Oxford, 1991)

${ }^{10}$ A. Messerer, R. Niessner, and U. Poschl, "Miniature pipe bundle heat exchanger for thermophoretic deposition of ultrafine soot aerosol particles at high flow velocities," Aerosol Sci. Technol. 38, 456 (2004).

${ }^{11} \mathrm{~J}$. C. Maxwell, "On stresses in rarified gases arising from inequalities of temperature," Philos. Trans. R. Soc. London 170, 231 (1879).

${ }^{12}$ P. S. Epstein, "Zur theorie des radiometers," Z. Phys. 54, 537 (1929).

${ }^{13} \mathrm{H}$. Brenner and J. R. Bielenberg, "A continuum approach to phoretic motions: Thermophoresis," Physica A 355, 251 (2005).

${ }^{14}$ C. F. Schadt and R. D. Cadle, "Thermal forces on aerosol particles," J. Phys. Chem. 65, 1689 (1961).

${ }^{15}$ J. R. Brock, "On the theory of thermal forces acting on aerosol particles," J. Colloid Sci. 17, 768 (1962).

${ }^{16}$ B. V. Derjaguin, Ya. I. Rabinovich, A. I. Storozhilova, and G. I. Shcherbina, "Measurement of the coefficient of thermal slip of gases and the thermophoresis velocity of large-size aerosol particles," J. Colloid Interface Sci. 57, 451 (1976).

${ }^{17} \mathrm{~W}$. $\mathrm{Li}$ and E. J. Davis, "Measurement of the thermophoretic force by electrodynamic levitation: Microspheres in air," J. Aerosol Sci. 26, 1063 (1995).

${ }^{18}$ L. Talbot, R. K. Cheng, R. W. Schefer, and D. R. Willis, "Thermophoresis of particles in heated boundary layer," J. Fluid Mech. 101, 737 (1980).
${ }^{19}$ C. Cercignani, Rarefied Gas Dynamics: From Basic Concepts to Actual Calculations (Cambridge University Press, Cambridge, 2000).

${ }^{20}$ Y. Sone, Kinetic Theory and Fluid Dynamics (Birkhauser, Boston, 2000).

${ }^{21}$ F. Sharipov and D. Kalempa, "Velocity slip and temperature jump coefficients for gaseous mixtures. I. Viscous slip coefficient," Phys. Fluids 15, 1800 (2003)

${ }^{22}$ F. Sharipov and D. Kalempa, "Velocity slip and temperature jump coefficients for gaseous mixtures. II. Thermal slip coefficient," Phys. Fluids 16, 759 (2004)

${ }^{23}$ N. J. McCormick, "Gas-surface accommodation coefficients from viscous slip and temperature jump coefficients," Phys. Fluids 17, 107104 (2005).

${ }^{24}$ B. E. Dahneke, "Slip correction factors for nonspherical bodies: Introduction and continuum flow," J. Aerosol Sci. 4, 139 (1973).

${ }^{25}$ M. L. Laucks, G. Roll, G. Schweiger, and E. J. Davis, "Physical and chemical (Raman) characterization of bioaerosols: Pollen," J. Aerosol Sci. 31, 307 (2000).

${ }^{26}$ L. D. Reed, "A continuum slip flow analysis of steady and transient thermophoresis," M.S. thesis, University of Illinois, Urbana-Champaign, Illinois (1971).

${ }^{27} \mathrm{H}$. J. Keh and H. J. Tu, "Thermophoresis and photophoresis of cylindrical particles," Colloids Surf., A 176, 213 (2001).

${ }^{28} \mathrm{~K}$. H. Leong, "Thermophoresis and diffusiophoresis of large aerosol particles of different shapes," J. Aerosol Sci. 15, 511 (1984).

${ }^{29}$ M. M. R. Williams, "Thermophoretic forces acting on a spheroid," J. Phys. D 19, 1631 (1986).

${ }^{30}$ H. J. Keh and C. L. Ou, "Thermophoresis of aerosol spheroids," Aerosol Sci. Technol. 38, 675 (2004).

${ }^{31}$ H. Lamb, Hydrodynamics (Dover, New York, 1945).

${ }^{32} \mathrm{H}$. J. Keh and J. L. Yu, "Migration of aerosol spheres under the combined action of thermophoretic and gravitational fields," Aerosol Sci. Technol. 22, 250 (1995).

${ }^{33} \mathrm{H}$. Brenner, "The Stokes resistance of a slightly deformed sphere," Chem. Eng. Sci. 19, 519 (1964).

${ }^{34}$ J. Happel and H. Brenner, Low Reynolds Number Hydrodynamics (Martinus Nijhoff, The Netherlands, 1983).

${ }^{35} \mathrm{~S}$. Haber and G. Hetsroni, "The dynamics of a deformable drop suspended in an unbounded Stokes flow," J. Fluid Mech. 49, 257 (1971).

${ }^{36}$ C. K. W. Tam and W. A. Hyman, "Transverse motion of an elastic sphere in a shear field," J. Fluid Mech. 59, 177 (1973).

${ }^{37} \mathrm{P}$. R. Wohl and S. I. Rubinow, "The transverse force on a drop in an unbounded parabolic flow,” J. Fluid Mech. 62, 185 (1974).

${ }^{38}$ S. Senchenko and H. J. Keh, "Slipping Stokes flow around a slightly deformed sphere," Phys. Fluids 18, 088104 (2006).

${ }^{39}$ F. A. Morrison, Jr., "Electrophoresis of a particle of arbitrary shape," J. Colloid Interface Sci. 34, 210 (1970).

${ }^{40}$ J. L. Anderson, "Colloid transport by interfacial forces," Annu. Rev. Fluid Mech. 21, 61 (1989). 